\title{
Weakly correlated triangular lattice metal $\mathbf{H}_{x} \mathrm{CoO}_{2}$ with $x \approx 0.3$
}

\author{
Masashige Onoda and Yuji Kikuchi \\ Institute of Physics, University of Tsukuba, Tennodai, Tsukuba 305-8571, Japan \\ E-mail: onoda@sakura.cc.tsukuba.ac.jp
}

\begin{abstract}
A new type of the triangular lattice system $\mathrm{H}_{x} \mathrm{CoO}_{2}(x \approx 0.3)$ is synthesized through a chemical extraction of $\mathrm{Na}$ from $\mathrm{Na}_{0.3} \mathrm{CoO}_{2}$ with $\mathrm{H}_{2} \mathrm{SO}_{4}$. The structure is modelled by two oxygen layers with a prismatic oxygen environment and a symmetric linear $\mathrm{H}-\mathrm{O}$ bond on the basis of the $\mathrm{x}$ ray powder diffraction and magic-angle spinning NMR analyses. Measurements of the electrical resistivity, thermoelectric power and magnetic susceptibility suggest a weakly correlated metallic state with the enhancement of effective mass. The transport anomalies, accompanied with the rapid decrease of thermoelectric power, are observed at about $10 \mathrm{~K}$.
\end{abstract}

PACS numbers: $61.10 . \mathrm{Nz}, 76.60 . \mathrm{Cq}, 72.80 . \mathrm{Ga}, 75.20 . \mathrm{En}$

\section{Introduction}

Transition-metal oxides have aroused considerable interest both from the basic science and the applied viewpoints. For various oxides with unfilled d orbitals which contain high- $T_{\mathrm{c}}$ superconductors, properties of correlated electron systems and quantum spinfluctuation systems have been investigated for a long time [1]. They are also utilized as several functional materials such as thermoelectric devices [2,3], rechargeable lithium batteries [3] and so on.

Since the proposal of resonating-valence-bond theory regarding a spin-liquid ground state for the triangular lattice system with Heisenberg interaction $[4,5]$, various oxide systems have been studied. The discovery of superconductivity in $\mathrm{Na}_{0.3} \mathrm{CoO}_{2} \cdot 1.3 \mathrm{H}_{2} \mathrm{O}[6]$ as well as the possible application to thermoelectric devices in the parent compound $\mathrm{Na}_{x} \mathrm{CoO}_{2}$ [2] throws up more interest for this system.

The $\mathrm{Na}_{x} \mathrm{CoO}_{2}$ system has several different phases with sheets of edge-sharing $\mathrm{CoO}_{6}$ octahedra interleaved by $\mathrm{Na}$ [7]. The stacking sequence of the oxygen layers leads to a number of sheets for a unit cell. Many works have dealt with the $\gamma$ phase that has a two-layer structure. This structure is basically described in terms of $\mathrm{CoO}_{6}$ octahedra which are joined by sharing edges to form a two-dimensional triangular lattice of Co ions [8]. Between these lattices, the $\mathrm{Na}$ atoms labelled $\mathrm{Na} 1$ and $\mathrm{Na} 2$ with trigonal prismatic environments reside in some occupation probability. According to the type of oxygen coordination for $\mathrm{Na}$ and the number of sheets, the $\gamma$ phase is expressed as 'P2'. The phase diagram of $\mathrm{Na}_{x} \mathrm{CoO}_{2}$ as a function of the $\mathrm{Na}$ concentration [9] indicates that, as $x$ increases from $\approx 0.3$ to $\approx 0.8$, the ground state goes from a normal 
metal to a Curie-Weiss metal through a poor metallic state at $x \approx 0.5$ accompanied by a partial valence order of Co [8].

The $\mathrm{Li}_{x} \mathrm{CoO}_{2}$ system has a triangular lattice similar to those of $\mathrm{Na}_{x} \mathrm{CoO}_{2}$ [10]. The structure of $\mathrm{LiCoO}_{2}$ is of the $\alpha-\mathrm{NaFeO}_{2}$ type, having the octahedral coordination for alkali atoms and three layers of oxygens (called 'O3'). This system has been investigated intensively from various viewpoints, since it is an excellent $\mathrm{Li}$ rechargeable battery. The crystal structures and the metal-insulator transitions in the $\mathrm{Li}$ de-intercalated or charged process are discussed in detail, especially relating to the performance of batteries. The end-member compound $\mathrm{CoO}_{2}$ prepared by electrochemical de-intercalation of $\mathrm{Li}$ for $\mathrm{LiCoO}_{2}$ has a $\mathrm{CdI}_{2}$-type 'O1' structure [11], while that synthesized soft-chemically has a 'P3' structure [12].

This article considers $\mathrm{H}_{x} \mathrm{CoO}_{2}(x \approx 0.3)$ derived from the nominal compound $\mathrm{Na}_{0.3} \mathrm{CoO}_{2}$ with soft-chemistry synthesis. While $\mathrm{HCoO}_{2}$ is known to have a 'P3' structure [13], neither the structure nor the electronic properties for the nonstoichiometric composition have been published. Details of the sample preparation and measurements are given in section 2. The structural model of $\mathrm{H}_{x} \mathrm{CoO}_{2}$ constructed with x-ray powder diffraction and magic-angle spinning (MAS) NMR is presented in section 3.1, and the electronic properties revealed through measurements of the electrical resistivity, thermoelectric power and magnetic susceptibility are described in section 3.2. Section 4 is devoted to conclusions.

\section{Experiments}

Polycrystalline specimens of $\mathrm{H}_{x} \mathrm{CoO}_{2}(x \approx 0.3)$ were synthesized by the soft-chemistry method as follows: first, nominal compounds of $\mathrm{Na}_{0.7} \mathrm{CoO}_{2}$ were made at $1073 \mathrm{~K}$ according to the procedure described in a previous work [14]. $\mathrm{Na}_{0.3} \mathrm{CoO}_{2}$ was prepared by mixing $10^{-3} \mathrm{~kg}$ of $\mathrm{Na}_{0.7} \mathrm{CoO}_{2}$ with 0.1 mol ${ }^{-1}$ of $\mathrm{Br}_{2}$ dissolved in acetonitrile in $\mathrm{Ar}$ through 4 days of stirring. The compounds obtained above $\left(9 \times 10^{-4} \mathrm{~kg}\right)$ were stirred in $2 \mathrm{~mol} \mathrm{l}^{-1}$ of $\mathrm{H}_{2} \mathrm{SO}_{4}$ for 6 days in air. Here, $\mathrm{H}_{2} \mathrm{SO}_{4}$ was repurified every other day. By washing them with water and drying in air, the powder specimens were finally obtained. For measurements of physical properties described below, the specimens were pressed into pellets at a pressure of $2.5 \times 10^{7} \mathrm{~N} \mathrm{~m}^{-2}$. A packing factor was about $85 \%$.

Inductively coupled plasma-optical emission spectroscopy (ICP) was done using a Nippon Jarrell-Ash ICAP-575 spectrometer. The MAS NMR spectra for the ${ }^{1} \mathrm{H}$ and ${ }^{23} \mathrm{Na}$ nuclei were recorded on Bruker Avance spectrometers under $14 \mathrm{~T}$ at $293 \mathrm{~K}$. Here, spinning speeds between 10 and $14 \mathrm{kHz}$ were used, and $\mathrm{H}$ and $\mathrm{Na}$ were referenced to the signals in silicones and $\mathrm{NaCl}$ solution at $0 \mathrm{ppm}$, respectively. An x-ray powder diffraction pattern was taken with $\mathrm{Cu} \mathrm{K} \alpha$ radiation at $293 \mathrm{~K}$ using a Rigaku RADIIC diffractometer. The four-terminal electrical resistivity and the thermoelectric power were measured with a dc method at temperatures between 4.2 and $300 \mathrm{~K}$. The magnetization measurements were performed at temperatures between 4.2 and $300 \mathrm{~K}$ by the Faraday method with a field of up to $1 \mathrm{~T}$. The magnetic susceptibility was deduced from the linear part of the magnetization-field curve with a decreasing field. 


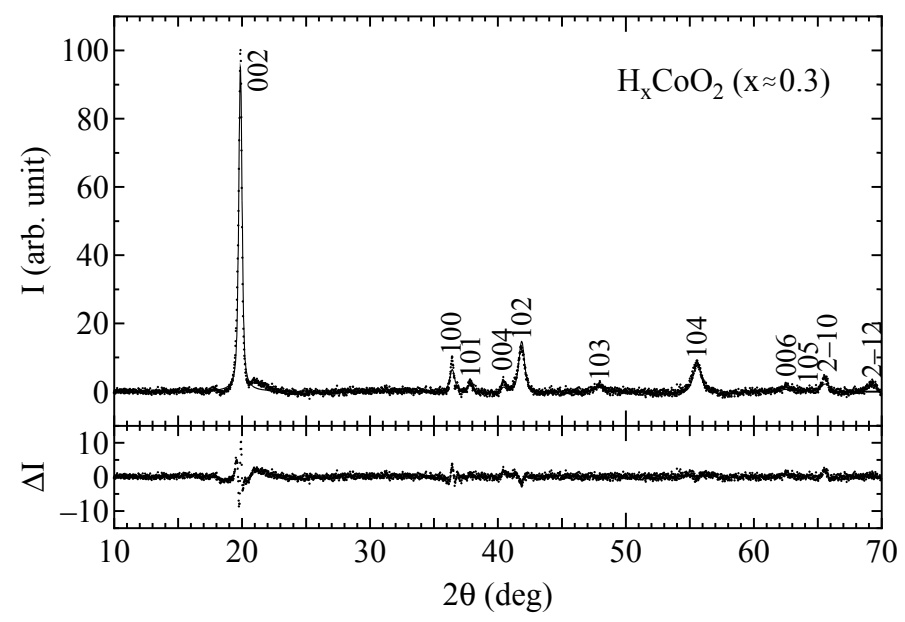

Figure 1. The x-ray powder diffraction pattern with $\mathrm{Cu} \mathrm{K} \alpha$ radiation at $293 \mathrm{~K}$ for the specimens derived through chemical extraction of $\mathrm{Na}$ from the nominal compound $\mathrm{Na}_{0.3} \mathrm{CoO}_{2}$ using $\mathrm{H}_{2} \mathrm{SO}_{4}$. The result in terms of Rietveld refinement is indicated by a full curve in the top panel and the difference between the observed and calculated intensities is plotted in the bottom panel.

\section{Results and discussions}

\subsection{Structural model}

ICP analysis indicates the specimen to have an Na concentration of $0.03(1)$ per Co ion. On the other hand, the MAS NMR analysis indicates that the Na signal does not appear, while the $\mathrm{H}$ signal having side bands of 20 ppm exists with a shift of $4.827(3)$ ppm and a full width at half maximum of $0.17(1) \mathrm{ppm}$ for $12 \mathrm{kHz}$ of spinning. Thus the $\mathrm{Na}$ ions detected with ICP may come from an impurity adhered to the surface of the specimens and a $\mathrm{HCl}$ solution. This idea is supported with the structure analysis described below.

Figure 1 indicates the x-ray powder diffraction pattern at $293 \mathrm{~K}$. The diffraction peaks are indexed as a hexagonal unit cell with dimensions $a=2.8434(4)$ and $c=8.909(3) \AA$. Note that a very weak reflection exists between $\left(\begin{array}{lll}1 & 0 & 0\end{array}\right)$ and $\left(\begin{array}{lll}1 & 0 & 1\end{array}\right)$, which is attributed to the peak from the minor phase of $\mathrm{Co}_{3} \mathrm{O}_{4}$. The $c$-axis is more than twice as large as that for the 'O1' structure of $\mathrm{CoO}_{2}$ with trigonal dimensions of $a=2.822(1)$ and $c=4.293(6) \AA$ [11], and it is much less than the value for the 'P2' structure of unmodulated crystal $\mathrm{Na}_{0.58} \mathrm{CoO}_{2}$ with hexagonal dimensions of $a=2.8180(5)$ and $c=11.005(9) \AA[8]$. On the basis of the Rietveld analysis [15] assuming the space group $P 6_{3} / m m c$, the structure is finally modelled with zero thermal factors for all of atoms as follows: the Co atom resides in $\left(\begin{array}{lll}0 & 0 & 0\end{array}\right)$ of the $2 \mathrm{a}$ site and the $\mathrm{O}$ atom is in $\left(\frac{2}{3} \frac{1}{3} 0.1035(18)\right)$ of the $4 \mathrm{f}$ site, corresponding to the 'P2' structure. The nearest neighbour $\mathrm{O}-\mathrm{O}$ distance between the layers is 2.61(2) $\AA$, which is much smaller than that for $\mathrm{Na}_{0.58} \mathrm{CoO}_{2}, 3.55 \AA$ [8]. Since the distance from the $2 \mathrm{~b}$ site $\left(\begin{array}{lll}0 & 0 & \frac{1}{4}\end{array}\right)$ and/or $2 \mathrm{c}$ site $\left(\begin{array}{lll}\frac{1}{3} & \frac{2}{3} & \frac{1}{4}\end{array}\right)$ expected for the $\mathrm{Na}$ positions in $\mathrm{Na}_{x} \mathrm{CoO}_{2}$ to $\mathrm{O}$ atoms is very short $(2.08 \AA)$ as compared with the ionic bond length [16], the Na atoms are considered to be absent, which is consistent with the calculated Fourier maps and the MAS NMR analyses. The $\mathrm{H}$ atoms are expected to be located 


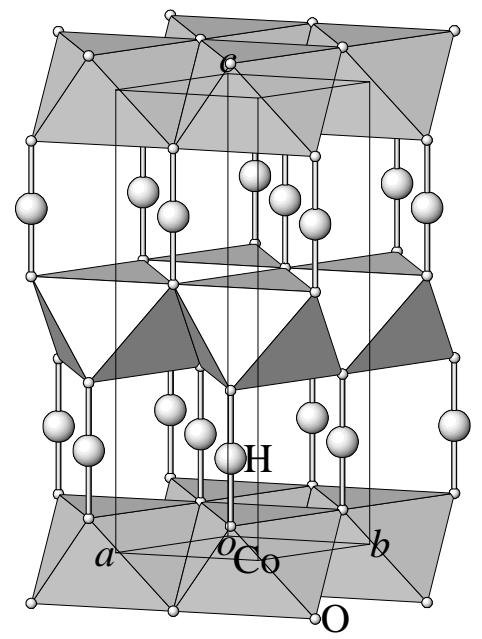

Figure 2. Clinographic view for the structural model of $\mathrm{H}_{x} \mathrm{CoO}_{2}(x \approx 0.3)$ with the octahedra for Co.

at the $2 \mathrm{~d}$ site of $\left(\frac{2}{3} \frac{1}{3} \frac{1}{4}\right)$ at an occupancy probability of about $\frac{1}{3}$ for the reason stated below. Here discrepancy factors are $R_{\mathrm{p}}=\sum\left|Y_{\mathrm{o}}-Y_{\mathrm{c}}\right| / \sum\left|Y_{\mathrm{o}}\right|=9.2 \%$ and $R_{\mathrm{wp}}=\left[\sum w\left(Y_{\mathrm{o}}-Y_{\mathrm{c}}\right)^{2} / \sum w Y_{\mathrm{o}}^{2}\right]^{1 / 2}=11.6 \%$, where $Y_{\mathrm{o}}$ and $Y_{\mathrm{c}}$ are the observed and calculated intensities of the pattern, respectively. According to the refinement for the two phases of $\mathrm{H}_{x} \mathrm{CoO}_{2}$ and $\mathrm{Co}_{3} \mathrm{O}_{4}$, the relative amount of the latter phase is about 1 \%. The clinographic view of the structural model of $\mathrm{H}_{x} \mathrm{CoO}_{2}(x \approx 0.3)$ is shown in figure 2 .

The Co ions are surrounded octahedrally with a $\mathrm{Co}-\mathrm{O}$ distance of $1.883(8)$ $\AA$. As shown in figure 2, there exist two triangular lattice layers of $\mathrm{CoO}_{2}$ linked by edge-shared $\mathrm{CoO}_{6}$ octahedra in the unit cell. The $\mathrm{H}$ atoms reside between the oxygen layers with a symmetric $\mathrm{H}-\mathrm{O}$ bond of $1.305(16) \AA$ that agrees well with the value for the two-coordinated $\mathrm{H}$ ion [16]. The $\mathrm{Co}-\mathrm{H}$ distance is $2.7669 \AA$, which is shorter than the $\mathrm{Co}-\mathrm{Co}$ distance in the $\mathrm{CoO}_{2}$ layer. The concentration of $\mathrm{H}$ may be estimated to be about $\frac{1}{3}$ with the condition that the Co valence calculated from the bond-lengths-bond-strength relation [17] agrees with that from the chemical formula assuming full occupancy of $\mathrm{O}$ ions. Thus the Co ion has a valence of $3 \frac{2}{3}$. In order to know detailed structural properties including the oxygen concentration, further investigations are necessary.

\subsection{Transport and magnetic properties}

The temperature dependence of the electrical resistivity $\rho$ for $\mathrm{H}_{x} \mathrm{CoO}_{2}(x \approx 0.3)$ is shown in figure 3(a). The resistivity increases gradually with decreasing temperature above $T_{\rho} \simeq 10 \mathrm{~K}$, below which it is almost constant as shown in the top panel of figure 3(c). The specimen is considered to be essentially metallic taking account of intergrain conductions due to the low packing factor as described in section 2. This behaviour is quite similar to that for a heavy fermion system $\mathrm{LiV}_{2} \mathrm{O}_{4}$ that exhibits a nonmetallic temperature dependence for loosely packed polycrystalline specimens [18] and indicates a metallic variation for the single-crystal specimens $[19,20]$. The data above $T_{\rho}$ appear to be expressed as a parallel combination of metallic resistivity $\left(\rho_{\mathrm{m}}\right)$ 

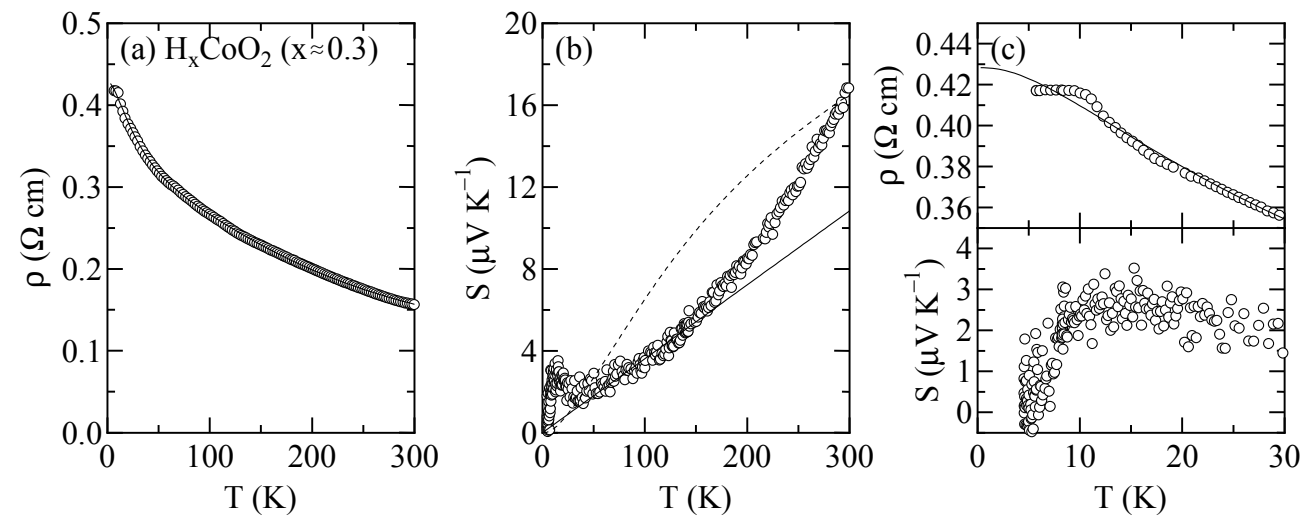

Figure 3. The temperature dependences of (a) the electrical resistivity and (b) the thermoelectric power for $\mathrm{H}_{x} \mathrm{CoO}_{2}(x \approx 0.3)$, and (c) their low-temperature parts. The full curves in (a) and (c) denote a fit to equation (1), and the full line and dotted curve in (b) are fits to equations (4) and (5), respectively, with parameters given in the text

with a power of $p$ and variable-range hopping (VRH) resistivity in three dimensions $\left(\rho_{\mathrm{v}}\right)$ for the loosely packed specimen $[21]$ :

$$
\rho=\left(1 / \rho_{\mathrm{m}}+1 / \rho_{\mathrm{v}}\right)^{-1},
$$

where the first and second terms are written as

$$
\begin{aligned}
& \rho_{\mathrm{m}}=\rho_{0}+A T^{p}, \\
& \rho_{\mathrm{v}}=B \exp \left[\left(T_{0} / T\right)^{1 / 4}\right],
\end{aligned}
$$

respectively, $\rho_{0}$ being the residual resistivity. The full curve in figure $3($ a) provides the following parameters: $\rho_{0}=0.4282(7) \Omega \mathrm{cm}, A=25(1) \mu \Omega \mathrm{cm} \mathrm{K}^{-p}$ with $p=2.05(1)$, $B=8.8(1) \mathrm{m} \Omega \mathrm{cm}$ and $T_{0}=2.18(5) \times 10^{4} \mathrm{~K}$. In the present case, it is meaningless to discuss the VRH parameters based on the detailed theory [22], because they significantly depend on a packing factor. On the other hand, the metallic resistivity is suggested to follow the Fermi-liquid relation as far as this model is assumed. The values of $\rho_{0}$ and $A$ much larger than those near the metal-insulator boundary for the perovskite system $[23,24]$ may be attributed to the loose packing of the specimen in addition to the correlation effect.

The thermoelectric power $S$, which is not significantly influenced by the intergrain transport, should reflect more intrinsic properties. Figure $3(\mathrm{~b})$ indicates the temperature variation of the thermoelectric power for $\mathrm{H}_{x} \mathrm{CoO}_{2}(x \approx 0.3)$. For the temperature region measured, the thermoelectric power is positive, indicating the carrier to be hole. On decreasing the temperature from $300 \mathrm{~K}$, the thermoelectric power decreases rapidly above $130 \mathrm{~K}$, below which it has a linear dependence on temperature above $30 \mathrm{~K}$ and exhibits a hump at $T_{S} \simeq 10 \mathrm{~K}$, and then it nearly goes to zero. This temperature dependence as well as the small magnitude (about $16 \mu \mathrm{V}$ $\mathrm{K}^{-1}$ at $300 \mathrm{~K}$ ) suggests that the compound is metallic, although the overall behaviour is different from that expected from the Fermi-liquid theory. Applying the relation

$$
S=\left.\frac{\pi^{2} k^{2} T}{3 e} \frac{\mathrm{d} \ln \sigma(E)}{\mathrm{d} E}\right|_{E_{\mathrm{F}}},
$$


where $k$ is the Boltzmann constant, $\sigma(E)$ is a conductivity-like function of electron energy $E$ with the form $\sigma(E) \propto E^{q}$ and $E_{\mathrm{F}}$ is the Fermi energy, to the data between 30 and $130 \mathrm{~K}, E_{\mathrm{F}}$ is estimated to be $7.1 \times 10^{3} q \mathrm{~K}$ on the basis of the full line in figure 3 (b). The exponent $q$ will be presented in section 4 . Considering that the parallel combination model of $\rho_{\mathrm{m}}$ and $\rho_{\mathrm{v}}$ is effective for this case, the thermoelectric power is simply written as

$$
S=\left(S_{\mathrm{m}} \rho_{\mathrm{v}}+S_{\mathrm{v}} \rho_{\mathrm{m}}\right) /\left(\rho_{\mathrm{m}}+\rho_{\mathrm{v}}\right),
$$

where $S_{\mathrm{m}}$ and $S_{\mathrm{v}}$ are from the paths related to $\rho_{\mathrm{m}}$ and $\rho_{\mathrm{v}}$, respectively. On the assumption that $S_{\mathrm{m}} \approx 0$ and $S_{\mathrm{v}} \propto T^{1 / 2}[21]$, a fit to the data at room temperature is obtained as shown by the dotted curve in figure $3(\mathrm{~b})$. The reason why the parallel combination model does not fit the data is that it corresponds to a limit for the two kinds of transport paths. Actually it is expected that the data points above $130 \mathrm{~K}$ exist between the two limits of Fermi-liquid theory (full line) and the parallel combination model (dotted curve). On the other hand, the Heikes formula for the case of $T, U \gg W$ but $T \ll U$ in the Hubbard model, where $U$ and $W$ are the Coulomb repulsion energy and the bandwidth, respectively, leads to $S=0$ for the Co valence of $3 \frac{2}{3}[25]$, which would also be consistent with the small value of $S$ taking account of the possible small difference of carrier density from the commensurate value. However, it may be not the case judging from the susceptibility results described below.

The magnetic susceptibility $\chi$ and its inverse of $\mathrm{H}_{x} \mathrm{CoO}_{2}(x \approx 0.3)$ as a function of temperature are shown in figures $4(\mathrm{a})$ and (b), respectively. Here the data containing the contribution from the slightly adsorbed oxygens are removed $\ddagger$. The temperature dependence is apparently expressed as

$$
\chi=C /\left(T+T_{\mathrm{W}}\right)+\chi_{0},
$$

where the first term is the Curie-Weiss susceptibility $\chi_{\mathrm{CW}}$ with the Curie constant $C$ and the Weiss temperature $T_{\mathrm{W}}$, and the second term is the temperature-independent component from the orbital and diamagnetic susceptibilities. A fit to equation (6) provides the following parameters: $C=0.0170(3) \mathrm{emu} \mathrm{K} \mathrm{mol}^{-1}, T_{\mathrm{W}}=17.5(7) \mathrm{K}$ and $\chi_{0}=2.74(1) \times 10^{-4} \mathrm{emu} \mathrm{mol}^{-1}$. The Curie constant obtained here is about 4 $\%$ of the value for $S=\frac{1}{2}$ and $g=2$, and the constant component is several times larger than the exact value known for another triangular lattice system $\mathrm{LiVO}_{2}$ below the spin-singlet trimerization temperature [26] apart from the difference between the electronic structures. This result is in striking contrast to those for the Curie-Weiss type metal phase of $\mathrm{Na}_{x} \mathrm{CoO}_{2}[9]$.

Assuming that the Curie-Weiss component comes from lattice imperfections and/or impurity phases, it should be considered that the large constant susceptibility includes a Pauli component from the metallic phase. For simplicity, using a freeelectron relationship, the Pauli susceptibility with a constant Stoner-type enhancement is described as

$$
\chi_{\mathrm{P}}=\frac{N \mu_{\mathrm{B}}^{2}}{k T} F_{1 / 2}^{\prime}(\xi) / F_{1 / 2}(\xi)
$$

where $N$ and $\mu_{\mathrm{B}}$ are the number of electrons and Bohr magneton, respectively, and $F_{1 / 2}(\xi)=\int x^{1 / 2} /[\exp (x-\xi)+1] \mathrm{d} x$ with $\xi=E_{\mathrm{F}} / k T[27]$. The susceptibility is then written as

$$
\chi=\chi_{\mathrm{P}}+\chi_{0}+\chi_{\mathrm{CW}}
$$

$\ddagger$ The oxygen desorption process for the magnetization equipment leads to a significant increase of the susceptibility at low temperatures likely due to the oxygen deficiency and/or the partial decomposition of specimen. 


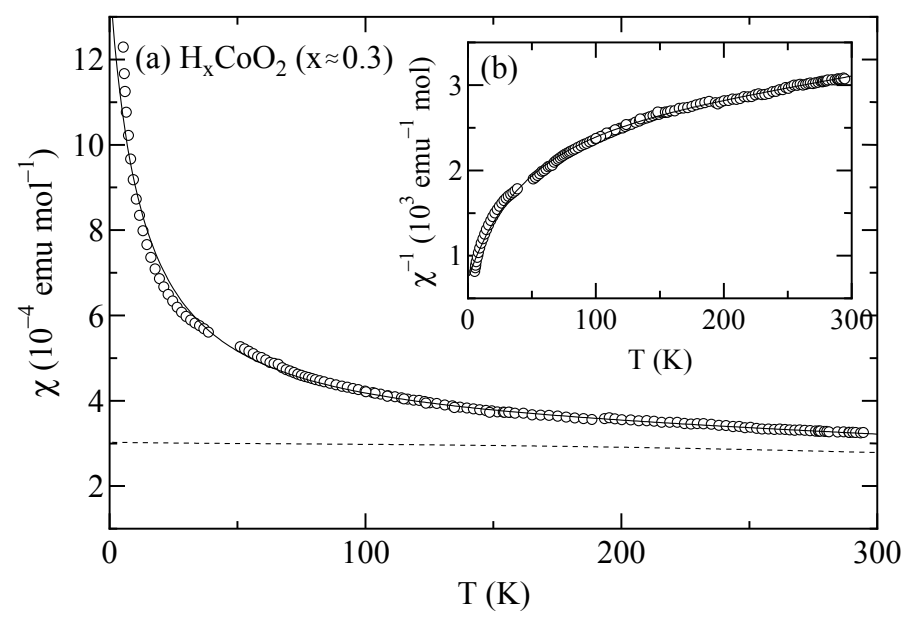

Figure 4. The temperature dependences of (a) the magnetic susceptibility and (b) its inverse for $\mathrm{H}_{x} \mathrm{CoO}_{2}(x \approx 0.3)$. The full curves in (a) and (b) denote fits to equation (8) with parameters given in the text, and the dotted curve in (a) indicates a contribution from the first and second terms in that equation.

The full curves in figures $4(\mathrm{a})$ and (b) provide the following results: $E_{\mathrm{F}}=1.17(4) \times 10^{3}$ $\mathrm{K}, \chi_{0}=-2(1) \times 10^{-5} \mathrm{emu} \mathrm{mol}^{-1}, C=0.0135(4) \mathrm{emu} \mathrm{K} \mathrm{mol}{ }^{-1}$ and $T_{\mathrm{W}}=12(1) \mathrm{K}$ for $N=\frac{2}{3}$ mol. The dotted curve in figure $4(\mathrm{a})$ indicates a contribution from $\chi_{\mathrm{P}}+\chi_{0}$. This $E_{\mathrm{F}}$ is rather smaller than that estimated using the correlated band local-density approximation $+U(\mathrm{LDA}+\mathrm{U})$ for $\mathrm{Na}_{1 / 3} \mathrm{CoO}_{2}[28]$ that is expected to have an electron concentration similar to that of the present compound: density of states at the Fermi level $\approx 2 \mathrm{eV}^{-1}$ and $E_{\mathrm{F}} \approx 5.8 \times 10^{3} \mathrm{~K}$. Thus the effective mass of carriers $m_{\mathrm{eff}}$ or $\chi_{\mathrm{P}}$ is enhanced by a factor of five over the free-electron values. The Landau diamagnetism expressed by $\chi_{\mathrm{L}}=-\frac{1}{3} \frac{m_{0}}{m_{\mathrm{eff}}} \chi_{\mathrm{P}}, m_{0}$ being a free-electron mass, is a minor contribution in this case. On the other hand, the analysis in terms of the spin- $\frac{1}{2}$ Heisenberg-type triangular lattice antiferromagnet [29] provides no reliable results, since a broad peak at certain temperature expected from this model does not appear in the data.

At temperatures $T_{\rho}$ and $T_{S}$, no any anomaly of the susceptibility is detected. Preliminary magnetization measurements cooled at zero field and $1 \mathrm{mT}$ indicate that the latter susceptibilities become larger than the former below $T_{\rho}$ and $T_{S}$. However, it is difficult to judge whether this property is really intrinsic or not due to the presence of the Curie-Weiss component.

\section{Conclusions}

A chemical extraction of $\mathrm{Na}$ from $\mathrm{Na}_{0.3} \mathrm{CoO}_{2}$ with $\mathrm{H}_{2} \mathrm{SO}_{4}$ provides a new type of the triangular lattice system $\mathrm{H}_{x} \mathrm{CoO}_{2}(x \approx 0.3)$. On the basis of the $\mathrm{x}$-ray powder diffraction and MAS NMR analyses, the structure is modelled by two oxygen layers with a prismatic oxygen environment ('P2' type) and a symmetric linear $\mathrm{H}-\mathrm{O}$ bond.

Due to the loose packing of specimens, the electrical resistivity seems to be explained by a parallel combination of the Fermi-liquid transport and VRH transport in three dimensions. The temperature dependence and the small magnitude of thermoelectric power may reflect metallic properties. The Heikes formula for the 
strongly correlated system also leads to the small magnitude of thermoelectric power, but this may not be the case. The magnetic properties are understood qualitatively with a mass-enhanced Pauli paramagnetism. From the results of thermoelectric power and magnetic susceptibility, the energy dependence of conductivity is considered to have the form of $\sigma(E) \propto E^{0.2}$. The hyperfine field in ${ }^{1} \mathrm{H}$ nuclei due to the Fermi contact interaction is also estimated to be $8.3 \mathrm{mT} \mu_{\mathrm{B}}^{-1}$. Putting the results obtained together, it may be concluded that the present compound is classified as a weakly correlated metal.

The mass enhancement of the present compound may be less significant than that of $\mathrm{Na}_{0.3} \mathrm{CoO}_{2}$ [9] from a comparison of their susceptibilities. This difference for $\mathrm{CoO}_{2}$ planes with the valence $\approx 3 \frac{2}{3}$ should be attributed to the coordinations of monovalent ions and/or the interlayer distances. The smaller correlation effects for the large electron concentration $\approx \frac{2}{3}$ suggests the $U / W$ ratio for this valence to be small likely due to the wide distribution of electrons for the $\mathrm{a}_{1 \mathrm{~g}}$ and $\mathrm{e}_{\mathrm{g}}$ bands. This reminds us of the difference in $U / W$ for the $3 \mathrm{~d}^{1}$ perovskite system: the $U / W$ for the cubic perovskite $\mathrm{SrVO}_{3}$ with degenerate orbitals is significantly smaller than that for the orthorhombic $\mathrm{LaTiO}_{3}$ with a singlet orbital due to Jahn-Teller distortion [30, 24]. It should be noted that the reduction of correlation effects for $\mathrm{Na}_{0.3} \mathrm{CoO}_{2}$ has recently been discussed using the LDA $+\mathrm{U}$ approach [31].

At $T_{\rho} \simeq T_{S} \simeq 10 \mathrm{~K}$, there exit transport anomalies accompanied with a plateau of resistivity and a rapid decrease of thermoelectric power.

\section{References}

[1] See, for example Imada M, Fujimori, A. and Tokura, Y 1998 Rev. Mod. Phys. 701039

[2] Terasaki I, Sasago Y and Uchinokura K 1997 Phys. Rev. B 56 R12685

[3] See, for a recent topic Onoda M and Onoda M 2006 Phys. Rev. B 73104108

[4] Anderson P W 1973 Mater. Res. Bull. 8153

[5] Fazekas P and Anderson P W 1974 Phil. Mag. 30423

[6] Takada K, Sakurai H, Takayama-Muromachi E, Izumi F, Dilanian R A and Sasaki T 2003 Nature 42253

[7] Fouassier C, Matejka G, Reau J-M and Hagenmuller P 1973 J. Solid State Chem. 6532

[8] Onoda M and Ikeda T 2007 J. Phys.: Condens. Matter 19186213 and references therein

[9] Foo M L, Wang Y, Watauchi S, Zandbergen H W, He T, Cava R J and Ong N P 2004 Phys. Rev. Lett. 92247001

[10] See, for example Antolini E 2004 Solid State Ionics 170159 and references therein

[11] Amatucci G G, Tarascon J M and Klein L C 1996 J. Electrochem. Soc. 1431114

[12] Venkatraman S and Manthiram A 2002 Chem. Mater. 143907

[13] Kondrashev Y D and Fedorova N N 1954 Dokl. Akad. Nauk SSSR 94229

[14] Ikeda T and Onoda M 2006 J. Phys.: Condens. Matter 188673

[15] Petricek V, Dusek M and Palatinus L 2000 Jana2000 The Crystallographic Computing System (Praha: Institute of Physics)

[16] Shannon R D 1976 Acta Crystallogr. A 32751

[17] Brese N E and O'Keeffe M 1991 Acta Crystallogr. B 47192

[18] Onoda M, Imai H, Amako Y and Nagasawa H 1997 Phys. Rev. B 563760

[19] Rogers D B, Gillson J L and Gier T E 1967 Solid State Commun. 5263

[20] Urano C, Nohara M, Kondo S, Sakai F, Takagi H, Shiraki T and Okubo T 2000 Phys. Rev. Lett. 851052

[21] Brenig W, Döhler G H and Heyszenau H 1973 Philos. Mag. 271093

[22] Onoda M 2004 J. Phys.: Condens. Matter 168957 and references therein

[23] Onoda M and Yasumoto M 1997 J. Phys.: Condens. Matter 95623

[24] Onoda M and Kohno M 1998 J. Phys.: Condens. Matter 101003

[25] Chaikin P M and Beni G 1976 Phys. Rev. B 13647

[26] Onoda M, Naka T and Nagasawa H 1991 J. Phys. Soc. Japan 602550 
[27] See, for example Wilson A H 1958 The Theory of Metals 2nd edn (London: Cambridge University Press)

[28] Lee K -W, Kuneš J and Pickett W E 2005 Phys. Rev. B 70045104

[29] Elstner N, Singh R R P and Young A P 1993 Phys. Rev. Lett. 711629

30] Onoda M, Ohta H and Nagasawa H 1991 Solid State Commun. 79281

[31] Lee K -W, Kuneš J, Novak P and Pickett W E 2005 Phys. Rev. Lett. 94026403 